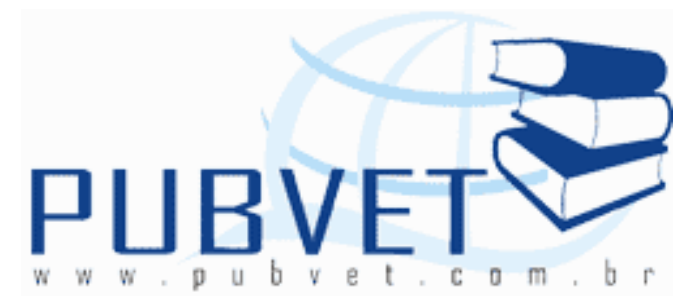

PUBVET, Publicações em Medicina Veterinária e Zootecnia.

\title{
Características qualitativas do filé mignon de cordeiros marinados com adição de glutamato de sódio
}

Luis Gustavo Castro Alves ${ }^{1}$, José Carlos da Silveira Osório², Maria Teresa Moreira Osório² ${ }^{2}$ Alexandre Rodrigo Mendes Fernandes ${ }^{3}$, Camila Magalhães da Cunha ${ }^{4}$, Thatiane da Cunha Cornélio ${ }^{4}$, Adriana Sathie Ozaki Hirata ${ }^{4}$, Ingrid Harumi de Souza Fuzikawa ${ }^{4}$

${ }^{1}$ Mestre em Zootecnia pela Universidade Federal da Grande Dourados, Dourados - MS, Email:gustavo353@hotmail.com;

${ }^{2}$ Professor (a) Visitante Nacional Sênior/CAPES/UFGD, Bolsista Produtividade CNPq;

${ }^{3}$ Professor do Curso e do Programa de Pós-Graduação em Zootecnia - UFGD;

${ }^{4}$ Mestranda do Programa de Pós-Graduação em Zootecnia - UFGD.

\section{Resumo}

Objetivou-se avaliar as características qualitativas do filé mignon de cordeiros submetidos ao processo de marinação com água e duas proporções de água + glutamato. Os filés $(n=32)$ foram submetidos à marinação e divididos em 4 tratamentos: sem água (controle), somente água, água $+5 \%$ glutamato, água $+10 \%$ glumatamato. Avaliou-se as características- peso inicial e final após o processo de marinação, capacidade de retenção de água (CRA), perda de peso por cozimento (PPC), força de cisalhamento (FC). Os tratamentos com glutamato $(5 \%$ e $10 \%)$ interferiram $(p<0,05)$ na diferença de peso da amostra 
ALVES, L.G.C. et al. Características qualitativas do filé mignon de cordeiros marinados com adição de glutamato de sódio. PUBVET, Londrina, V. 7, N. 26, Ed. 249, Art. 1644, Suplemento 2, 2013.

com maior peso após o processo de marinação. O tratamento controle interferiu $(p<0,01)$ na capacidade de retenção de água (CRA) com $(79,61 \%)$ contra os tratamentos com água $(67,93 \%)$, água + glutamato $5 \%(70,25 \%)$ e água + glutamato $10 \%(70,20 \%)$. Em relação à perda de peso por cozimento (PPC) os tratamentos com água $(33,50 \%)$, água + glutamato $5 \%(33,27 \%)$ e água + glutamato $10 \%(29,69 \%)$ interferiram $(\mathrm{p}<0,01)$ sobre 0 tratamento controle $(22,64 \%)$. A força de cisalhamento foi influenciada $(p<0,05)$, sendo que 0 tratamento controle e os tratamentos com água + glutamato apresentaram menores valores, 2,13 kgf, 2,09 kgf, 2,06 kgf, respectivamente, frente ao tratamento com água, 2,70 kgf. A utilização da marinação com soluções de água + glutamato melhora as características qualitativas do filé mignon de cordeiros.

Palavras-chave: carne, imersão, maciez, marinação, ovinos, suculência

\section{Qualitative characteristics of lamb loin marinated with addition of sodium glutamate}

\section{Abstract}

This study aimed to assess the quality of the filet mignon of lambs subjected to marination process with water and two amounts of glutamate + water solution. The filet $(n=32)$ underwent marination and divided into 4 treatments: no water (control), only water, water $+5 \%$ glutamato, water $+10 \%$ glumatamato. We evaluated the characteristics, weight initial and final after the marination process, water holding capacity (WHC), cooking weight loss (PPC), shear force $(F C)$. The treatments with glutamate $(5 \%$ and $10 \%)$ interfere $(p$ $<0.05$ ) in the weight difference of the sample weight after more marination process. The control interfered $(p<0.01)$ in the water-holding capacity (WHC) of $(79.61 \%)$ against treatments with water $(67.93 \%)$ and treatments with water $+5 \%$ glutamate $(7025 \%)$ and water $+10 \%$ glutamate $(70.20 \%)$. Regarding the cooking weight loss (PPC) The treatments with water (33.50\%) and treatments with water $+5 \%$ glutamate $(33.27 \%)$ and water $+10 \%$ 
glutamate $(29.69 \%)$ interfere $(p<0.01)$ over the control $8(22.64 \%)$. The shear force was influenced $(p<0.05)$, treatment and control and water + glutamate ( $5 \%$ e $10 \%$ ) were lower, $2.13 \mathrm{kgf}, 2.09 \mathrm{kgf}, 2.06 \mathrm{kgf}$, respectively, compared to treatment with water $2.70 \mathrm{kgf}$. The use of marination with water + glutamate improves the quality characteristics of lamb filet mignon.

Keywords: immersion, juiciness, marination, meat, sheep, tenderness

\section{INTRODUÇÃO}

A carne, porção comestível, está sendo cada vez mais valorizada em todos os seus aspectos, principalmente, pela sua qualidade nutricional e funcional, reforçando assim a necessidade do desenvolvimento de novas tecnologias pósabate que proporcionem características desejáveis à carne ovina e que possam ser utilizadas em escala comercial (Costa et al., 2008; Osório et al., 2013).

As indústrias produtoras de carne têm diversificado o processamento do seu produto final, dentre eles à produção de carnes marinadas, sendo que o processamento pode substituir as carnes tradicionais in natura no varejo. A técnica de marinação contribui para diferenciar sabor, melhorar textura e aumentar a conservação da carne. As vantagens deste método é a simplicidade, baixo custo, além de permitir produção de pequenos lotes. Um dos métodos de marinação é o de imersão, um procedimento antigo que consiste em submergir a carne em solução de água com adição de compostos ligantes como o sal, fosfatos e outros ingredientes (Daguer et al., 2010; Pastore 2006; Lemos, 2005; Xargayó, 2001).

O glutamato é um dos ingredientes empregados pela indústria de alimentos, conhecido por ter a capacidade de realçar outros compostos ativos que conferem sabor. Em sua forma livre, é detectado por receptores gustativos e proporciona o quinto gosto básico "umami", siginifica "saboroso" (Jinap e Hajeb, 2010). A forma mais comum de sua utilização é através do glutamato monossódico (sigla em inglês MSG- monosodium glutamate), que é o sal 
sódico do aminoácido ácido glutâmico, sendo obtido através da fermentação de açúcares, principalmente oriundos da cana de açúcar e milho (Sano, 2009).

Nesse cenário, faz-se necessário oferecer alternativas de técnicas de processamento e ingredientes que possam influenciar nas características preconizadas pelos consumidores como sabor, maciez, suculência. Sendo assim objetivou-se avaliar as características qualitativas do filé mignon de cordeiros submetidos ao processo de marinação com água e duas proporções de água + glutamato.

\section{MATERIAL E MÉTODOS}

As análises foram realizadas no Laboratório de Análises de Produtos Agropecuários - UFGD, utilizou-se 32 filés mignon (músculo Psoas major) de cordeiros, sendo distribuídos em um delineamento inteiramente casualizado, com 4 tratamentos e 8 repetições. Foram compostos pelo tratamento controle (sem adição de água e solução), tratamento com somente água, tratamento com água e solução de $5 \%$ de glutamato e tratamento com água e solução de $10 \%$ de glutamato. A proporção de água foi calculada em relação ao peso total das amostras de cada tratamento na razão de $2: 1$. A partir da quantidade total de água de cada tratamento foi calculada a quantidade do glutamato, $5 \%$ ou 10\% (Tabela 1).

Tabela 1 - Esquema de distribuição das amostras de filé de cordeiro em relação ao peso total das amostras de cada tratamento e a determinação da quantidade de água e glutamato.

\begin{tabular}{ccccc}
\hline Grupo & $\mathrm{N}$ & $\begin{array}{c}\text { Peso Total das } \\
\text { Amostras }(\mathrm{g})\end{array}$ & $\begin{array}{c}\text { Água } \\
(\mathrm{ml})^{*}\end{array}$ & $\begin{array}{c}\text { Glutamato } \\
(\mathrm{g})^{* *}\end{array}$ \\
\hline Controle & 8 & 166 & - & - \\
Água & 8 & 190 & 380 & - \\
Água $+5 \%$ Glutamato & 8 & 186 & 372 & 18 \\
Água $+10 \%$ Glutamato & 8 & 178 & 356 & 35 \\
\hline
\end{tabular}

*Quantidade de água: peso total das amostras de cada tratamento (2:1) $* *(5 \%$ de $372=18$ e $10 \%$ de $356=35)$ 
ALVES, L.G.C. et al. Características qualitativas do filé mignon de cordeiros marinados com adição de glutamato de sódio. PUBVET, Londrina, V. 7, N. 26, Ed. 249, Art. 1644, Suplemento 2, 2013.

As amostras dos filés foram colocadas individualmente em copo plástico descartável e a quantidade de água foi dividida proporcionalmente para que cada amostra recebesse a mesma quantidade da solução. A justificativa para a utilização de um recipiente individual para cada amostra foi em decorrência de que se o volume da solução (água + glutamato) ou somente água fosse ligado ao recipiente aberto às amostras não teriam o contato completo com o meio liquido (Alves et al., 2012). Posteriormente as mesmas foram armazenadas em geladeira a temperatura de $10^{\circ} \mathrm{C}$ pelo período de 1 hora para sofrerem o processo de marinação.

Em seguida foram realizadas as análises qualitativas nas amostras, para a determinação da capacidade de retenção de água (CRA), foram retiradas aproximadamente 2 gramas do músculo Psoas major e colocadas entre papel filtro, isolaram-se as partes inferiores e superiores entre duas placas de vidro e mantidas sob um peso de $5 \mathrm{~kg}$ durante 5 minutos. Posteriormente, as amostras foram pesadas e, por diferença, calculou-se a quantidade perdida. $\mathrm{O}$ resultado foi expresso em porcentagem de água exsudada em relação ao peso inicial da amostra, conforme descrito por Cañeque e Sañudo (2000).

$\mathrm{Na}$ análise de perda de peso por cozimento (PPC) as amostras foram assadas em forno elétrico até atingirem temperatura superior a $70^{\circ} \mathrm{C}$ no seu centro geométrico. Os pesos antes e depois de cozimento foram utilizados para os cálculos das perdas totais (Pardi et al., 2001).

A análise da força de cisalhamento (FC) foi determinada utilizando-se as mesmas amostras de perda de peso ao cozimento para a análise de maciez objetiva. Foram retirados 2 cilindros por amostras de músculo totalizando 16 cilindros por tratamento. Os cilindros foram retirados no sentido da fibra (livre de gordura e tecido conjuntivo) utilizando-se vazador com $1,3 \mathrm{~cm}$ de diâmetro através de manuseio manual. A técnica foi registrada, pelo aparelho Texture Analyser TA.XT.plus, acoplado a lâmina Warner Bratzler, com objetivo de determinar a força necessária para cortar transversalmente cada cilindro. Após foi calculada a média de força de corte dos cilindros para representar a força 
de cisalhamento de cada amostra expressa em kgf, metodologia proposta por AMSA (1995).

Os resultados foram submetidos á análise de variância e quando significativo os dados foram submetidos ao teste de Tukey a $1 \%$ e $5 \%$ de probabilidade com auxílio do programa SAEG.

\section{RESULTADOS E DISCUSSÃO}

Os valores dos pesos das amostras em relação ao peso inicial e final e a diferença após o processo de marinação encontram-se na tabela 2. 0 tratamento controle por não sofrer adição de água no processo de marinação não foi incluído para esta avaliação, em virtude de que os demais tratamentos sofreram contato no meio liquido.

Tabela 2- Média do peso inicial e final das amostras de filé e a diferença de peso após a adição de água ou de solução (água+ glutamato) nas proporções de $5 \%$ e $10 \%$ no processo de marinação.

\begin{tabular}{|c|c|c|c|c|c|}
\hline \multirow[b]{2}{*}{ Características } & \multicolumn{4}{|c|}{ Tratamentos } & \multirow[b]{2}{*}{ CV } \\
\hline & Água & $\begin{array}{c}5 \% \\
\text { Glutamato }\end{array}$ & $\begin{array}{c}10 \% \\
\text { Glutamato }\end{array}$ & $\mathrm{F}$ & \\
\hline Peso Inicial(g) & 23,808 & 23,330 & 22,268 & Ns & 39,09 \\
\hline Peso Final ( $\mathrm{g}$ ) & 25,284 & 25,752 & 24,788 & Ns & 37,91 \\
\hline Diferença Peso $(\mathrm{g})$ & $1,476 b$ & $2,422 a$ & $2,519 a$ & * & 33,80 \\
\hline
\end{tabular}

Ns - não significativo * Significativo $5 \%$

Os tratamentos que receberam adição de solução com glutamato (5\% e $10 \%$ ) obtiveram maior diferença de peso quando comparado ao tratamento que recebeu somente água. Isto ocorreu em decorrência da maior concentração de sólidos dissolvidos pelos tratamentos com 5\% e $10 \%$ de solução com glutamato, permitindo a migração do maior volume de água e dos solutos para o interior do músculo devido à polaridade das miofibrilas, mesmo que as camadas do tecido conjuntivo (epimísio, perimísio, endomísio) atuem como uma barreira de migração destes solutos (Lemos, 2001).

Mesmo o componente sódio sendo um dos elementos principais neste processo, o conteúdo de sódio (em percentual de massa) no glutamato é 
ALVES, L.G.C. et al. Características qualitativas do filé mignon de cordeiros marinados com adição de glutamato de sódio. PUBVET, Londrina, V. 7, N. 26, Ed. 249, Art. 1644, Suplemento 2, 2013.

aproximadamente 3 vezes mais baixo do que no cloreto do sódio (sal de cozinha), $12 \%$ e $39 \%$, respectivamente (Yamaguchi e Takahashi, 1984). As proporções utilizadas na solução com glutamato ( $5 \%$ ou $10 \%$ ) neste experimento sobre o volume total de água para cada tratamento ficou bem próxima ao resultado encontrado por Alves et al. (2012) que utilizaram a marinação de filés mignon com cloreto de sódio (sal de cozinha). De acordo com Smith e Young (2007) o principal fator que dita à efetividade do processo de marinação quanto ao rendimento é a quantidade da salmoura penetrada, sendo esta, altamente relacionada às propriedades funcionais dos aditivos adicionados.

Tabela 3 - Médias das características qualitativas do músculo Psoas major submetidos ao processo de marinação

\begin{tabular}{|c|c|c|c|c|c|c|}
\hline \multirow[b]{2}{*}{ Características } & \multicolumn{4}{|c|}{ Tratamentos } & \multirow[b]{2}{*}{$\mathrm{F}$} & \multirow[b]{2}{*}{ CV } \\
\hline & Controle & Água & $\begin{array}{c}5 \% \\
\text { Glutamato }\end{array}$ & $\begin{array}{c}10 \% \\
\text { Glutamato }\end{array}$ & & \\
\hline CRA (\%) & $79,61 a$ & $67,93 b$ & $70,25 b$ & $70,20 b$ & $* *$ & 4,81 \\
\hline PPC (\%) & $22,64 b$ & $33,50 a$ & $33,27 a$ & $29,69 a$ & $* *$ & 12,51 \\
\hline FC (kgf) & $2,13 b$ & $2,70 a$ & $2,09 b$ & $2,06 \mathrm{~b}$ & $*$ & 21,59 \\
\hline
\end{tabular}

* Significativo 5\%** Significativo $1 \%$

CRA- Capacidade de Retenção Água; PPC - Perda de Peso ao Cozimento; FC Força de Cisalhamento.

De acordo com Osório et al. (2009) a capacidade de retenção de água é parâmetro biofísico-químico que poderia definir como o maior ou menor nível de fixação de água de composição do músculo. Na Tabela 3, o tratamento controle diferiu significativamente tanto do tratamento com água quanto dos tratamentos com a solução de água + glutamato ( $5 \%$ e 10\%). Este efeito pode estar associado pelo fato do contato das amostras com o meio liquido e, atuações de seus compostos fizeram com que o exsudado das células saísse após a aplicação de força durante a análise quando comparado ao grupo controle, uma vez que, Silva Sobrinho et al. (2005) e (Lemos, 2005) afirmam que esta avaliação consiste na habilidade de retenção de água durante a aplicação de força ou tratamento externos. 
Os valores da perda de peso por cozimento foram inversamente aos obtidos na capacidade de retenção de água, ou seja, os maiores valores encontrados para os tratamentos com água e com soluções com água + glutamato ( $5 \%$ e $10 \%$ ), as amostras que na capacidade de retenção de água reteram menos exsudado perderam mais exsudado para esta avaliação interferindo no rendimento do corte, corroborando com Bressan et al. (2001) em que a perda de peso por cozimento é influenciada pela capacidade de retenção de água nas estruturas da carne.

Em relação aos resultados obtidos para força de cisalhamento foi verificado diferença significativa entre os tratamentos, o tratamento com água diferiu dos tratamentos controle e das soluções de água + glutamato (5\% e $10 \%$ ), mesmo Pardi et al. (2001) sugerindo que o corte filé mignon possui acentuada maciez em decorrência de pequena ou ausência de tecido conjuntivo e colágeno.

$\mathrm{Na}$ prática os valores encontrados para a força de cisalhamento das amostras dos tratamentos certamente não seriam detectados pelos consumidores, já que são valores muito próximos entre eles, e somente o equipamento (texturômetro) conseguiu identificar e, estão próximos aos valores encontrados por Cezar e Souza (2007) em torno de 2,27 kgf e foram consideradas como macias. Entretanto, há necessidade de se testar a utilização de níveis crescentes de solução com glutamato em cortes que possam sofrer maiores influências nas características qualitativas e, além de ampliar os dados na literatura sobre este assunto.

\section{CONCLUSÃO}

A utilização da marinação com soluções de água + glutamato melhora as características qualitativas do filé mignon de cordeiros 
ALVES, L.G.C. et al. Características qualitativas do filé mignon de cordeiros marinados com adição de glutamato de sódio. PUBVET, Londrina, V. 7, N. 26, Ed. 249, Art. 1644, Suplemento 2, 2013.

\section{AGRADECIMENTOS}

Aos grupos de pesquisa Ovinotecnia e de Carcaças e Carnes da UFGD

\section{REFERÊNCIAS BIBLIOGRÁFICAS}

AMSA- AMERICAN MEAT SCIENCE ASSOCITION. Research guidelines for cokkery sensory and instrumental tenderness measurement of fresh meat. Chicago, p.48, 1995.

ALVES, L.G.C.; OSÓRIO, M.T.M.; NUBIATO, K.E.Z.; CUNHA, C.M.; CORNÉLIO, T.C.; RODRIGUES, G.C.G.; COLOMBO, J.B.; FERNANDES, A.R.M. Utilização da marinação em filé de cordeiro. In: VI Congresso de Ciências Agrárias da Unioeste, 2012, Marechal Cândido Rondon. Anais do VI Congresso de Ciências Agrárias da Unioeste, 2012.

BRESSAN, C.; PRADO, O.V.; PÉREZ, J.R.O.; LEMOS, A.L.S.C.E.; BONAGURIO, S. Efeito do peso ao abate de cordeiros Santa Inês e Bergamácia sobre as características físico-químicas da carne. Ciência e Tecnologia de Alimentos, Campinas, 21(3): p.293-303, 2001.

CAÑEQUE, V.; SAÑUDO, C. Metodologia para el estúdio de la calidad de la canal y de la carne em ruminantes. INIA. Madrid. 254 pp. 2000.

CEZAR, M.F.; SOUSA, W.H. Carcaças ovinas e caprinas: obtenção, avaliação e classificação. Uberaba: AgropecuáriaTropical, 232 pp., 2007.

COSTA, R.G.; CARTAXO, F.Q.; SANTOS, N.M., QUEIROGA, R.C.R.E. Carne caprina e ovina: composição lipídica e características sensoriais. Revista Brasileira de Saúde e Produção Animal, v.9, n.3, p.497-506, 2008.

JINAP, S.; HAJEB, P. Glutamate. Its applications in food And contribution to health. Appetite. v.55, n.1, p.1-10. 2010.

DAGUER, H.; ASSIS, M.T.Q.M.; BERSOT, L.S. Controle da utilização de ingredientes não cárneos para injeção e marinação de carnes. Ciência Rural, v.40, n.9, p. 2037-2046, 2010.

LEMOS, A.L.S.C. Marinação - satisfazendo o consumidor e agregando valor à carne de aves. TecnoCarnes CTC,11(2): p.1-4, 2001.

LEMOS, A.L.S.C. Tecnologias disponíveis para realçar a maciez da carne bovina. In: Congresso Brasileiro de Ciência e Tecnologia de Carnes, 3., São Pedro. Anais.... São Pedro, 2005.

OSÓRIO, J.C.S.; OSÓRIO, M.T.M. Produção de carne ovina: Técnicas de avaliação "in vivo" e na carcaça. 2a ed. Pelotas: Universidade Federal de Pelotas. Ed. Universitária, p.25-32, 2005.

OSÓRIO, J.C.S.; OSÓRIO, M.T.M.; SAÑUDO, C. Características sensoriais da carne ovina. R. Bras. Zootec., (supl. especial), v.38, p.292-300, 2009.

OSÓRIO, J.C.S.; OSÓRIO, M.T.M.; VARGAS JUNIOR, F.M.; FERNANDES, A.R.M.; SENO, L.O. Avaliação da carcaça em animais de produção. Org. Jaqueline Schneider Lemes e Victor Fernando Buttow Roll. Pelotas. Ed. Carta, Cap. 1, p.13-30, 2013.

PARDI, M.C; SANTOS, I.F. SOUZA, E.R; PARDI, H.S. Ciência, higiene e tecnologia da carne: tecnologia da sua obtenção e transformação. Goiânia: Centro Editorial e Gráfico Universidade de Goiás, v. 1, 586 pp., 1993.

PARDI, M.C.; SANTOS, I.F.; SOUZA, E.R. Ciência, higiene e tecnologia da carne. 2.ed. Goiânia:UFG, 623 pp., 2001. 
PASTORE, G.M. O crescimento da indústria de carnes a partir de produtos com valor agregado. Jornal UNICAMP, Campinas, v.317, p.2, mar, 2006.

SANO, C. History of glutamate production. The American Journal Clinical Nutrition, 90: 728S32S. 2009.

SAÑUDO, C. Analisis sensorial - Calidad organoléptica de la carne. In: Curso Internacional de Analise Sensorial de Carne e Produtos Cárneos, 2004, Pelotas. Anais... Pelotas, p.45-68, 2004.

SILVA SOBRINHO, A.G.; PURCHAS, R.W.; KADIM, I.T.; YAMAMOTO, S.M. Características de qualidade da carne de ovinos de diferentes genótipos e idades ao abate. Revista Brasileira de Zootecnia, 34(3), p.1070-1078, 2005.

SMITH, D.P.; YOUNG, L.L. Marination pressure and phosphate effects on broiler breast fillet yield, tenderness, and color. Poultry Science, Ithaca, v.86, p.2666-2670, 2007.

XARGAYÓ, M. Marination of fresh meats by means of spray effect. Fleischwirtschaft International, v.2, n.1, p.70-74, 2001.

YAMAGUCHI, S.; TAKAHASHI, C. "Interactions of monosodium glutamate and sodium chloride on saltiness and palatability of a clear soup". Journal of food Science 49 (1), p.82-85, 1984. 